\title{
Modified Radical Hysterectomy
}

National Cancer Institute

\section{Source}

National Cancer Institute. Modified Radical Hysterectomy. NCI Thesaurus. Code

C136238.

The surgical removal of the uterus and surrounding structures, including bilateral pelvic lymph nodes, the parametrium, and the upper one-third to one-half of the vagina. The amount of parametria and uterosacral lig ament removed with the uterus is less than that removed during a radical hysterectomy. 